\title{
Incidence of non-cardiac morbidity in patients indergoing compolex cardiac sugery
}

\author{
Babulal Sethia
}

$\mathrm{D}$ R HAGEMO AND HIS COLLEAGUES ARE TO BE congratulated there report in this issue of the journal report, in which they highlight the incidence of non-cardiac morbidity in the context of patients treated for hypoplastic left heart syndrome during the 1990's. The incidence of non-cardiac morbidity in patients undergoing complex cardiac surgery in the neonatal period has been the focus of investigation by other groups interested specifically in the conduct of cardiopulmonary bypass. The debate concerning the relative merits of bypass with continuous low flow versus circulatory arrest remains to be resolved. It would have been helpful had information been provided concerning the protocols used for cardiopulmonary bypass, although it is clearly beyond the specific remit of the investigation. This information could be of importance, nonetheless, since the reported mortality (16 out of 31 children) is much higher than that currently expected in the management of these patients by experienced teams.

The authors have noted the incidence of some dysmorphic characteristics, and even microcephaly in some of the patients undergoing surgery. They do not state whether there were any patients excluded from consideration for this particular type of palliative therapy. This is important because, in the current era, it is no longer necessary

Accepted for publication 21 March 1997 in the majority of cases to commence surgery on an emergency basis. This permits time for formal evaluation of the child and, if necessary, exclusion on the basis of significant coexisting morbidity of non-cardiac origin. In our own experience in Birmingham, we have often counselled parents against pursuing a Norwood type of palliation when we have anticipated significant coexisting neurological dysfunction.

Notwithstanding these comments, the Norwegian experience suggests that only 3 out of 29 patients ( 2 being excluded because they declined follow-up evaluation) experienced a satisfactory outcome in the early or intermediate term. This finding in itself reinforces the importance of careful systematic evaluation of patients being considered for this complex type of therapy, and also of instituting careful follow-up in order to evaluate long term quality of life. This approach will also need to be evaluated in the context of the totality of neonatal and infant cardiac surgery. Only in this way can we determine whether the deficits observed by the authors are specific to patients with hypoplastic left heart syndrome or whether they possibly represent morbidity attendant upon the current perfusion techniques as utilised in the very young patients undergoing cardiac surgery.

Birmingham Children's Hospital, Birmingham, UK 